\title{
Fabrication of elastomer pillar arrays with elasticity gradient for cells migration, elongation and patterning
}

\author{
Bin Wang ${ }^{\text {a }}$, Jin Wei ${ }^{\text {a }}$, Xiaolong Tu ${ }^{\text {a }}$, Jian Shi ${ }^{\text {a }}$, Yong Chen ${ }^{\text {a,b* }}$ \\ ${ }^{a}$ PASTEUR, Département de chimie, école normale supérieure, PSL \\ University, Sorbonne Université, CNRS, 75005 Paris, France \\ ${ }^{b}$ Institute for interdisciplinary research center, Jianghan University, Wuhan 430056, China \\ * Corresponding author. Email: yong.chen@ens.fr (Y. Chen)
}

Abstract:

The elasticity of the cell and that of the supporting ECM in tissue are correlated. In some cases, the modulus of the ECM varies with a high spatial gradient. To study the effect of such a modulus gradient on the cell culture behavior, we proposed a novel yet straightforward method to fabricate elastomeric pillar substrates with different height gradients, which could provide a large range of elasticity gradient from $2.4 \mathrm{kPa}$ to $60 \mathrm{kPa}$. The micropillars were integrated into a microfluidic chip to demonstrate the elasticity variation, with the theoretical results proving that the elasticity of the two micropillar substrates was in the same range whereas with distinguished gradient strengths. Fibroblast seeded on the micropillar substrates showed migration toward stiffer area but their elongation highly depended on the strength of the elasticity gradient. In the case of high gradient strength, cells could easily migrate to the stiffer area and then elongated perpendicularly to their migration direction. Otherwise, cells were mostly elongated in the direction of the gradient. Our results also showed that when the cell density was sufficiently high, cells tend to be oriented in the same direction locally which was affected by both underneath pillars and cell-cell contact. The elasticity gradients could also be generated in ripple-shape and the cells behaviors showed the feasibility of using the micropillars for cell patterning applications. Moreover, the gradient pillars substrates were further used for the aggregates formation of induced pluripotent stem cells (iPSCs), thus providing an alternative substrate to study the effect of substrate elasticity on stem cell behavior and differentiation.

Keywords: Elastomer micropillars, Elasticity gradient, Cell culture, Migration 


\section{Introduction}

Most cells in tissues are embedded in extracellular matrices (ECM) and interacting with other cells to maintain their morphology and well-defined functions [1-3]. Depending on the tissue type, the elasticity of the cell and the ECM varies correlatively over a large range from $0.1 \mathrm{kPa}$ for the soft tissues like brain to $10 \mathrm{kPa}$ even larger for the stiff tissues like muscles and bones. Though the correlation between the cell and ECM mechanic properties can be easily understood by considering the request of cytoskeletal development as well as other cell functions such as cell proliferation, migration, differentiation and apoptosis [4-8], it is still challenging to study the elasticity dependent cell-ECM interaction because of the lack of reliable material models and the fact that the most of studies were carried out with glass, plastic surfaces or synthetic polymers having a elasticity several order larger than that of the natural ECM. For this reason, hydrogels and elastomeric substrates were developed as soft and elasticity adjustable substrates for cell culture studies [9-11].

Hydrogel and elastomer have also been used to create elasticity gradients for cell migration [12, 13] and interfacial tissues studies [14]. The main methods so far used for control the stiffness of biomaterial substrates include adjusting material amount, crosslinking density and photopolymeriztion time [15-18]. Moreover, hydrogels like polyacrylamide was used to create substrate with stiffness gradient[20]. In such cases, altered cross-linker amount impacts not only bulk mechanics but also molecular-scale material properties including porosity, surface chemistry, and backbone flexibility and binding properties of immobilized adhesive ligands. Furthermore, these substrates are often limited to simple patterns and small stiffness gradients.

More recently, advances in micro- and nanofabrication methods are enabling researchers to develop sophisticated substrates and scaffolds. Micropillar arrays with uniform height fabricated by lithography techniques have been used to study the relationship between traction forces and focal adhesion development, cell morphology in response to substrate rigidity and migration [21-23], providing new insights into the underlying mechanisms of mechanosensing. Pillar arrays with elasticity gradient have also been proposed by continuous changing the pillar size or introducing a stepped micropillar array device $[24,25]$. These approaches are intuitive but it is more interesting to achieve a higher elasticity gradient by continuously changing the pillar height since the elasticity of 
the pillar is more critically dependent on the pillar height than the pillar size or pillar spacing, and pillars with the same size and the space would facilitate the final analyses due to the same cell adhesion properties. Typically, the elastomeric pillars were made in polydimethylsiloxane (PDMS) which have a Young's module of $2 \mathrm{MPa}$, while PDMS pillar arrays with $2 \mu \mathrm{m}$ diameter and $4 \mu \mathrm{m}$ pitch could have an effective surface modulus of $4.6 \mathrm{kPa}$ and $53.68 \mathrm{kPa}$ when the height of the pillar arrays was $9 \mu \mathrm{m}$ and $4 \mu \mathrm{m}$, respectively [23]. Thus, by solely changing the pillar height it would be possible to cover a broad range of the substrate elasticity requested for ECM elasticity studies.

In this work, we present a simple yet straightforward method to fabricate dense pillar arrays with elasticity gradient by continuously varying the pillar height, achieving by creating high density elastomer pillar arrays with uniform top surface but different pillar heights on the same substrate. The current study makes further progress by examining how the gradient strength, developed by modulating the wavy period, notably affects cells behaviors. Moreover, we showed that such pillar arrays were reliable to control aggregates formation of induced pluripotent stem cells (iPSCs).

\section{Materials and methods}

\subsection{Fabrication of the substrate}

PDMS micropillar arrays with height gradient were prepared by two-step photolithography and soft lithography, as schematically described in figure S1. Briefly, hole arrays with diameter $2 \mu \mathrm{m}$ and period $5.5 \mu \mathrm{m}$ were firstly patterned on a blank Cr mask pre-coated with AZ 1518 photoresist (Nanofilm, Wetlake Village, California) by direct laser writing (DLW) using a $\mu$ PG101 system (Heidelberg Instruments, Germany). After development and chrome-etch, the mask was spin coated with $15 \mu \mathrm{m}$ thick SU-8 3010 resist (Microchem, USA) at a speed of $2000 \mathrm{rpm}$ for $30 \mathrm{~s}$. This resist layer was then exposed with another mask with $10 \mu \mathrm{m}$ linewidth and $160 \mu \mathrm{m}$ or $310 \mu \mathrm{m}$ pitch-size absorber stripes. After development, the sample was spin-coated with $7 \mu \mathrm{m}$ thick SU-8 3005 at a speed of $2000 \mathrm{rpm}$ and UV exposed without mask, resulted in a wavy pattern due to the relative small thickness of the coating resist with respect to the SU-8 3010 stripes. Next, the sample was coated with another $7 \mu \mathrm{m}$ thick SU-8 3005 at $2000 \mathrm{rpm}$ for $30 \mathrm{~s}$ and UV exposed from back side, resulted in SU-8 micropillar arrays with height gradients and uniform top surfaces. 
The fabricated SU-8 features arrays were used as mold (master) for PDMS casting after exposed to trimethylsilanechloride (TMCS) vapor for $30 \mathrm{~min}$. A pre-polymer solution of PDMS was prepared at a ratio of base polymer to cross-liker 10:1 (GE RTV 615 kit). After casting, the sample was placed in a vacuum chamber to remove air bubbles and then cured in an oven of $80^{\circ} \mathrm{C}$ for $2 \mathrm{~h}$. After peelingoff, this PDMS negative-tone replica of the master was treated by oxygen plasma for $90 \mathrm{~s}$ and TMCS evaporation for $1 \mathrm{~h}$ before second PDMS casting which was performed in the similar way. Finally, PDMS micropillar arrays with height gradients and uniform top surfaces were obtained, as shown in figure 1.

\subsection{Microfluidic device integration}

The micropillars with height gradient were integrated into a microfluidic channel to demonstrate the elasticity gradient of pillars. Briefly, a thin layer of PDMS patterned with micropillar arrays was bonded to a glass slide after treated with plasma for 2 min. The top PDMS layer containing a channel with width $6 \mathrm{~mm}$ and height $20 \mu \mathrm{m}$ was produced by soft lithography. First, a chromium mask with designed parameter was produced and then replicated into a photoresist layer (SU8-3010). After the development and evaporation of TMCS, PDMS was poured on the SU8 mold and cured at $80{ }^{\circ} \mathrm{C}$ for 2 h. After peeling off, holes of the inlet and outlet were punched. The surfaces of upper and bottom PDMS layers were treated by plasma for $2 \mathrm{~min}$, following by a thermal bonding at $80{ }^{\circ} \mathrm{C}$ for $10 \mathrm{~min}$.

To demonstrate height-dependent elasticity of pillars, a method based on gas-liquid interfacial tension was introduced, as illustrated in figure 2(a). Briefly, deionized water was firstly loaded into the device, and then the gas (air) was introduced into the channel, the flow was controlled using a multichannel pressure generator (Fluidic controller FC-PVL-II, Mesobiosys), which was perpendicular to gradient direction. The air-liquid interface was controlled at the first row of micropillar arrays by adapting the applied pressure. The images of micropillars before and after deformation were recorded, and the displacements of the pillars were determined by the shift of the top surface.

\subsection{Cell culture}

PDMS samples were sterilized with a UV light overnight. The top surfaces of the pillars were then functionalized by micro-contact printing as described below. Firstly, a flat PDMS layer was immersed 
in a solution of $50 \mu \mathrm{g} \mathrm{ml}^{-1}$ fibronectin (Sigma-Aldrich, France) in distilled water for $1 \mathrm{~h}$. After washing three times in distilled water and blowing with dry under nitrogen, this flat PDMS layer was placed in conformal contact with the top surfaces of the PDMS pillars for $15 \mathrm{~min}$. Before that, the sample was treated by oxygen plasma for $3 \mathrm{~min}$ to facilitate the fibronectin transfer from the flat PDMS layer to the PDMS pillar substrate.

NIH 3 T3 cells were cultured in DMEM (Gibco) supplemented with 10\% fetal bovine serum and 1\% penicillin and streptomycin, 1\% L-glutamine and 1/10,000 fungizone (Sigma-Aldrich, France). Before seeding, the cells were dissociated with $0.05 \%$ Trypsin EDTA solution at $3^{\circ} \mathrm{C}$ for $3 \mathrm{~min}$. After centrifugation, they were re-suspended in the medium with a concentration of about $20,000 \mathrm{cells} \mathrm{ml}^{-1}$. The PDMS pillar samples were placed in 6-well plate and $4 \mathrm{ml}$ cell suspension was dropped in each well. Immediately after seeding, the entire system was moved to an incubator $\left(37^{\circ} \mathrm{C}, 5 \% \mathrm{CO}_{2}\right)$ for cell culture. Finally, the culture medium was changed every other day.

IPSCs (episomal line from Life Technologies) were cultured with complete E8 medium in a culture dish coated with 1: 100 diluted vitronectin at $37{ }^{\circ} \mathrm{C}$ in $5 \% \mathrm{CO}_{2}$. The medium was renewed every day until the cells reached about $80 \%$ confluence. Cells were then collected by dissociation using a $0.5 \mu \mathrm{M}$ EDTA DPBS solution. Before cell seeding, the micropillar substrates were sterilized with a UV light overnight and the top surfaces of the pillars were functionalized with vitronectin by microcontact printing. After that, iPSCs with density of $2 \times 10^{5}$ in $50 \mu \mathrm{L}$ of E8 medium containing $10 \mu \mathrm{M}$ ROCK inhibitor (Y-27632) were seeded on the surface of the substrates placed in the plate, then transferred into an incubator for cell attachment for $1 \mathrm{~h}$. Afterward, $2 \mathrm{~mL}$ of fresh E8 medium containing $10 \mu \mathrm{M}$ ROCK inhibitor were added to the culture. Then, the culture medium was replaced by E8 medium without ROCK inhibitor everyday.

\subsection{Scanning electronic microscopy}

The high-resolution images for cell on oblique micropillars were obtained with a scanning electron microscope (Hitachi S3030) operated at $10 \mathrm{kV}$. Before observation, the samples were firstly fixed for $30 \mathrm{~min}$ in PBS containing 4\% formaldehyde. Then, the samples were rinsed twice with PBS buffer, and immersed in 30\% ethanol (in DI) for $30 \mathrm{~min}$. After that, the samples were dehydrated in a graded series of ethanol: $50 \%, 70 \%, 80 \%, 90 \%$ and $100 \%$ every $10 \mathrm{~min}$ for two times, followed by 
nitrogen critical point drying. Finally, a thin film of gold of $10 \mathrm{~nm}$ thickness was deposited on the samples by sputter Auto500 (Germany) for SEM observation.

\subsection{Immunofluorescent staining}

The samples were rinsed by PBS to remove detached and dead cells. Then, cells were fixed with $4 \%$ formaldehyde for $30 \mathrm{~min}$ and permeabilized with in $0.5 \%$ Triton-X-100 for $10 \mathrm{~min}$ at room temperature. Next, cells incubated in a blocking solution containing $0.1 \%$ TX and 3\% BSA for 30 min. After wash with PBS, skeleton and nuclear of the cells were stained with Phalloidin-FITC and DAPI (Sigma, Deisenhofen, Germany) respectively at the same time for $20 \mathrm{~min}$ at room temperature.

For iPSCs, cells were incubated with primary antibodies after blocking, i.e., anti-OCT4 (1: 100 diluted, Life Technologies), anti-SSEA4 (1: 100 diluted, Life Technologies) in blocking buffer overnight at $4{ }^{\circ} \mathrm{C}$. After incubation with the primary antibody overnight at $4{ }^{\circ} \mathrm{C}$, cells were treated with secondary antibodies, i.e., Alexa Fluor 488 Donkey anti-rabbit IgG (1:300 diluted) and Alexa Fluor 594 Donkey anti-mouse IgG (1:300 diluted) both from Santa Cruz in blocking buffer at room temperature for $1 \mathrm{~h}$. For nuclei staining, cells were incubated with $300 \mathrm{ng} / \mathrm{mL}$ of DAPI for $30 \mathrm{~min}$, and finally washed three times with PBS. The stained cells were observed under a LSM Zeiss 710 confocal microscope.

The Image J software (open source image analysis software, Version 1.41) was introduced to analyze the fluorescence images of cells. To evaluate alignment, the cell nucleus was fitted with an ellipse. From the ellipses, the major and minor axes, and angle between the major axis and the reference direction (micropillars height gradient direction) were then computed. The alignment angles and anisotropy (ratio of the major axis to minor axes) were calculated accordingly, three samples and over 80 cells in total in each group were analyzed.

\subsection{Statistical analysis}

Statistical analyses were performed by GraphPad Prism software. Quantitative findings are presented as mean \pm standard deviation. Student's t-test was used to compare differences between two experimental groups and oneway analysis of variance (ANOVA) with Tukey post-hoc test was used to make pairwise comparisons between multiple groups. Statistical significance was set to $p<0.05$. 


\section{Results and discussion}

\subsection{Characterization of the substrate}

The proposed culture substrates made of micro-pillars with continuous variation of height were fabricated by photolithography techniques. The critical step of the fabrication of such pillar arrays is the formation of the sloped stripes. Here, we first fabricated $15 \mu \mathrm{m}$ height narrow stripes using standard SU8 pattern technique and then spin-coating $7 \mu \mathrm{m}$ thick SU-8 3005 resist, which gave rise to concave structures due to relatively low viscosity of the resist. For the same reason, spin-coating of another layer of 3005 resist on such a non-plan substrate resulted in a flat surface. After backside UV exposure through the Cr mask and development, pillar arrays with height variation could be worked out. After twice PDMS casting, positive-tone elastomeric pillars could be obtained with uniform top surfaces but different heights on which different cell morphologies can be observed due to the elasticity difference of underneath substrate, as depicted in figure 1(a). Figure 1(b)-(d) show scanning electron microscopy (SEM) images of the PDMS pillars of $2 \mu \mathrm{m}$ diameter and periodic height variation of $160 \mu \mathrm{m}$ (P160) and $310 \mu \mathrm{m}(\mathrm{P} 310)$ periods.

To demonstrate the elasticity variety of the micropillars, a method based on pressure-driven displacements of the micropillars in microfluidic channel was illustrated, as schemed in figure 2(a). Firstly a liquid phase was introduced into the channel, followed by air introducing. The air-liquid interface was controlled in the first row of the pillars. Figure 2(b) and 2(c) show images of micropillars before and after displacement in P160, it could be seen that the interface was arcuate because of the different displacements of the pillars (figure 2(f)). Similarly, the P310 samples showed similar curve after deformation of the pillars (figure 2(d), 2(e) and 2(h)). So with the same pressure the displacements of the pillars were different, qualitatively proving that the micropillar arrays with gradient height resulted in rigidity variation. Moreover, it could be seen that the displacements in P160 and P310 were almost in the same range, from $0.03 \mu \mathrm{m}$ to $1.73 \mu \mathrm{m}$, but the variation strength was different.

The elasticity of the micropillars was further quantified based on Euler-Bernoulli beam theory [26], the spring constant of the pillars can be calculated by $k=3 \pi E r^{4} / H^{3}$, where $E$ is the Young's modulus of the bulk PDMS, $r$ and $H$ are the radius and height of the pillar, respectively. Assuming a pillar diameter of $2 \mu \mathrm{m}$ and a Young's module of bulk PDMS of $2 \mathrm{MPa}$, we obtain a spring constant of 
$77.87 \pm 7.86$ and $4.07 \pm 0.11 \mathrm{nN} / \mu \mathrm{m}$ for a PDMS pillar with height of $3.79 \pm 0.12$ and $10.13 \pm 0.14$ $\mu \mathrm{m}$, respectively. Considering our dense pillar arrays as continuous elastic substrates, an equivalent Young's modulus can be deduced using $E_{e}=9 k / 4 \pi a$, where $a$ is the characteristic length representing the pillar radius or the mean size of focal adhesion for a continuous substrate [27]. Accordingly, the variation of the effective Young's modulus of PDMS pillar samples P160 and P310 can be visualized (figure 2(g) and 2(i)), suggesting a modulus change of almost 30 times from the softer $(2.4 \mathrm{KPa})$ to the harder $(60.0 \mathrm{KPa})$ areas in half period.

\subsection{Cell motility and cell migration}

NIH 3T3 cells are commonly used to study interaction of fibroblasts with culture substrates [10, 28]. They were chosen also because of their comparable Young's modulus (in the range of 3 to $12 \mathrm{kPa}$ ) with respect to that of our PDMS pillar samples [29]. Figure 3(a) shows snapshots of time-lapse image series of the cells on pillars of sample P160. After attachment, the cells began to spread and sense the surrounding environment. For those firstly attached on the soft area, they trend to migrate to the stiff area. For those already located on the stiff area, they maintained in the same area. Afterward, they spread, showing different migration behavior (movie S1) in different pillar areas. Note also that the fibroblasts on stiff substrates have relatively large spreading area comparing to that on soft substrates which have in general a rounded phenotype with prominent microvilli [30-32]. While in our case, the cells firstly attached on soft areas spread out to shorter pillar areas by mechanotransduction effect. Hela cells were also tested on sample P160, showing neither elongation nor migration caused by elasticity gradient of PDMS pillars (Fig. S1) due to fact that they have different Young's module (in the range of 12 to $35 \mathrm{kPa}$ ) [29].

Figure 3(b) and 3(c) compare the distribution and the morphology of the cells on P160 and P310 samples after incubation for $24 \mathrm{~h}$. On sample P160, most of the cells were found in the stiff area, whereas on sample P310, cells were more homogenously distributed. More interesting, cells are elongated along the stiff stripes on P160 but the direction of elasticity gradient on P310. The travelling distance of the cells on two types of substrates were plotted in figure 3(d) and 3(e), showing an average travelling length of $59.33 \pm 2.30 \mu \mathrm{m}(21.45 \pm 1.53 \mu \mathrm{m})$ along the elasticity gradient and $10.29 \pm 3.74$ $\mu \mathrm{m}(11.34 \pm 3.21)$ in the perpendicular direction on sample P160 (P310), respectively. Accordingly, 
we obtained an average traveling speed of $3.23 \pm 0.26 \mu \mathrm{m} \mathrm{h}^{-1}\left(1.38 \pm 0.19 \mu \mathrm{m} \mathrm{h}^{-1}\right)$ along the elasticity gradient for sample P160 (P310), while no significant difference in travelling speed was observed in the perpendicular direction (figure 3(f)). These results suggest that not only the elasticity but also the elasticity gradient affect the morphology and migration of NIH 3T3 cells as well as their distribution on the patterned surfaces. Apparently, a high stiff gradient causes a fast cell migration but a low elasticity gradient enables more effectively cell elongation.

\subsection{Cell proliferation and cell morphology}

Figure 4 shows immunofluorescence images of NIH 3T3 cells on flat PDMS, PDMS pillar arrays P160 and P310 after incubation for 1 and 4 days. Clearly, cells were randomly distributed on the flat PDMS sample without preferential elongation at day 1 . However, after culture for 4 days, the cell density became high and the cell-cell interaction became important so that they appeared to elongated in parallel to each other in small and even large zone. On sample P160, most of the cells were elongated perpendicular to the elasticity gradient and preferentially located in the stiff area on day 1 but the same effect of parallel elongated, not necessarily along the same direction after incubation for 4 days. As time proceeds, fibroblast, as spindle-shaped cells, prefer to organize well-aligned nematic or polar domains when confluent in topological defects, which is also influenced by cell-substrate interaction $[33,34]$. In this case, with the cell density increased via cell proliferation, the cells already stayed on short pillar areas gradually freeze the proliferated cells movement, possibly resulted in the proliferated cells remained elongated along the gradient direction and actively aligned with their neighbors in a nematic or polar phase, whereas there was still around $10 \%$ cells showed $80-90^{\circ}$ alignment angle at day 4 due probably to the initially migrated cells. On sample P310, cells were strongly elongated parallel to the elasticity gradient, in contrast to that on sample P160, and more cells are preferentially located in the stiff areas on day 1. On the other hand, cells seeded on micropillar arrays with uniform height showed random morphology (figure S4), confirming again the striking effect of the elasticity gradient. As can be seen, the cell length along the elongated axis of the cells on P160 is larger than half of the elasticity period so that cells were subjected to a strong elasticity gradient which leads them to migrate to the stiffer area and to change their orientation. On sample P310, the average elasticity of the substrate is smaller than that of P160 so the cells are elongated or stretched to power their stress 
filament. After incubation for 4 days on sample P310, highly parallel cell elongation was observed due to relatively strong cell-cell interaction and already stretched cell morphology.

Figure 5(a) and 5(b) shows more clearly the actin-filament (green) and nuclei (blue) of the cells on samples P160 and P310 after incubation for 1 day. Similar cells morphology was confirmed by SEM images (figure 5(c) and 5(d)) from which it can be clearly seen that the elastomer PDMS pillars were deformed by cells traction. The statistical data of the analyses are reported in figure 5(e)-(h). Here, the shape anisotropy is defined as the ratio of the length to the width of the cells and their nuclei and only images of the cells after 1 day incubation were used for size and anisotropy analyses because of easy image processing. Interestingly, cells cultured on flat PDMS have large spreading and nucleus areas to because of large elasticity ( $\sim 2 \mathrm{MPa}$ ) comparing to the PDMS pillar substrates (figure 5(e) and 5(f)). The shape anisotropy of the cells on sample P310 is significantly larger than that on sample P160 but the nucleus shape anisotropy on both samples is larger than that on plat PDMS (figure 5(g) and (h)).

In general, cells sense the elasticity of the substrate through integrin complexes, leading to the earliest focal adhesions formation and then undergo elasticity dependent conformational changes with F-actin and RhoA-mediated contractile force generation $[35,36]$. When the traction forces located on both sides of cells are large enough and well-balanced, cells will have no directional migration. When the underlying matrix has a elasticity gradient, the misbalanced traction forces will induce a polarization of the actin fibers toward the stiffer part [37-39] Previously, studies of cells on stepped micropillar arrays have already shown that cells migrated across the soft-stiff boundary toward the stiffer area due to the strong difference of the traction forces in two sides of boundary. The possible reason for this phenomenon was that a faster force increases and higher saturated force values on stiffer substrates and an increasing stress with respect to substrate stiffness, After that, polarized cells coming from a softer substrate will suddenly exert large traction forces as they touch the stiff side, and thus rotate to migrate perpendicularly to the stiff substrate [22]. Our results suggest that the mechanotranduction of the cells depends on the elasticity gradient of the substrate. On sample P160 with a large elasticity gradient $(0.68 \mathrm{KPa} / \mu \mathrm{m})$, the difference in contracile force between two extremes of the cell in the direction parellel to the elasticity gradient could be large enough to guide cell migration. Once 
entring in the stiff area, cells explore rigid part of the pillar arrays, giving rise to elongation perpendicular to the elasticity gradient. On sample P310 with a small elasticity gradient $(0.39 \mathrm{KPa} / \mu \mathrm{m})$, cells explore longer distance along in the direction parallel to the elasticity gradient but the difference in contracile force between two extremes of the cell might not be sufficient to stimulate the migration so that the motility of the cells is less remarkable and the cell morphology remained mostly elongated.

\subsection{Cell patterning and aggregation}

Other types of wavy structures can also be fabricated so that different elasticity gradients of pillar substrates can be generated. Figure 6 shows a ripple-like design of PDMS pillar substrate using the same pillar parameters and $160 \mu \mathrm{m}$ wavy-period (figure 6(a) and 6(b)), the calculated effective Young's module (figure 6(c)) and NIH 3T3 cells cultured on the pillar substrate after incubation for 1 day, 3 days and 4 days (figure 6(d)-(f)). As expected, cells were mainly localized in the stiffer areas and elongated in the direction perpendicular to the elasticity gradient during the first 3 days culture. With the increase of the cell density, the orientation of elongated cells was perturbed for the same reason discussed before. Furthermore, we demonstrated that the micropillar substrate P160 could be also used for iPSCs aggregation formation, as shown in figure 7(a). At day 0, the iPSCs homogeneously attached on micropillars while after 1 day culture, the cells aggregated to the stiff areas, the aggregations then started to expand. To evaluate the pluripotency of the iPSC colonies, pluripotent stem cell markers OCT4 and SSEA4 were used to stain the colonies formed on both pillar substrates and monolayer cells on flat PDMS as a control after 2 days culture. The immunofluorescence images show clearly that the expression of these markers was maintained (figure 7(b)). From the quantitative data (figure 7(c) and 7(d)), the expression of the markers on both substrates was comparable, showing above $90 \%$ cells were positive, which is in agreement with other studies that the micropillar substrates could be used to expand and differentiate iPSCs $[21,40]$. Heretofore in literatures, it's been emphasized that embryoid bodies-like aggregates formation is critical for pluripotency maintaining and subsequent differentiation of pluripotent stem cells [41, 42], and previously we also demonstrated embryonic body-like aggregations formation on micropillar substrates with different elasticity with the help of a flexible stencil for cardiac differentiation[43], here we made a significant progress in building elsaticity gradient in one substrate and successfully obtained the colonies, due probably to the elasticity gradient 
induced migration. The gradient micropillar substrates could be further used to study how the gradient elasticity affect stem cell differentiation. Moreover, iPSCs cultured on micropillar substrates could benefit from the enhanced diffusion underneath the iPSC colonies and the 3D feature of the scaffold for decreased cell-matrix traction [21]. These features make gradient pillar substrates useful alternatives for studies of pluripotency maintenance and for the controlled differentiation of iPSCs on different substrates.

\section{Conclusion}

We have proposed a simple yet straightforward method to fabricate PDMS pillar arrays of $2 \mu \mathrm{m}$ diameter, $5.5 \mu \mathrm{m}$ pitch and 3.8 to $10.2 \mu \mathrm{m}$ height with a uniform top surface, which were used as culture substrates with an effective surface elasticity varying between $2.4 \mathrm{KPa}$ to $60.0 \mathrm{KPa}$. NIH 3T3 cells were chosen to demonstrate the elasticity gradient dependent cell morphology and cell migration. Our results show that cells were effectively elongated and preferentially located on the stiff areas but the cell elongation was more pronounced on the soft pillar arrays. With the increase of cell density, the interaction between cells became more and more important which forces parallel cell alignment on both soft and stiff areas, which led to a highly stretched cell morphology and highly parallel cell alignment of the NIT 373 cells on the pillar array with a relative small elasticity gradient (P310) but

only a short-distance alignment correlation of the cells on the pillar array with a relative large elasticity gradient (P160). Furthermore, the iPSCs on gradient micropillars showed similar migration behavior and formed aggregates on stiffer areas. Because of their outstanding mechanical and geometric properties, elastomeric micropillar substrates might be potential candidates for more systematic studies of iPSCs culturing, expansion, and induced differentiation.

\section{Acknowledgements}

This work was supported by Agence de Recherche Nationale under contract No ANR-13-NANO0011-01 (Pillarcell) and ANR-17-CE09-0017 (AlveolusMi- mics). This work was also supported by the Cost action BIONECA (CA 16122) of European Commission, the DIM ELICIT program of Ilede-France, and the Pre- maturation projects of PSL Valorization. 


\section{References}

[1] Wang N and Ingber D E 1994 Control of cytoskeletal mechanics by extracellular matrix, cell shape, and mechanical tension Biophys. J. 66 2181-9

[2] Hynes R O 2009 The extracellular matrix: not just pretty fibrils Science 326 1216-9

[3] Sun Y, Chen C S and Fu J 2012 Forcing stem cells to behave: a biophysical perspective of the cellular microenvironment Ann. Rev. Biophys. 41 519-42

[4] Wozniak M A and Chen C S 2009 Mechanotransduction in development: a growing role for contractility Nat. Rev. Mol. Cell Bio. 1034

[5] Kim P, Yuan A, Nam K-H, Jiao A and Kim D-H 2014 Fabrication of poly (ethylene glycol): gelatin methacrylate composite nanostructures with tunable stiffness and degradation for vascular tissue engineering Biofabrication 6024112

[6] Bao Y, Huang Y, Lam M L, Xu T, Zhu N, Guo Z, Cui X, Lam R H and Chen T-H 2016 Substrate Stiffness Regulates the Development of Left-Right Asymmetry in Cell Orientation ACS Appl. Mater. Interfaces 8 17976-86

[7] Zhong Y and Ji B 2013 Impact of cell shape on cell migration behavior on elastic substrate Biofabrication 5015011

[8] Castillo A B and Jacobs C R 2010 Mesenchymal stem cell mechanobiology Curr. Osteoporos. Rep. 8 98-104

[9] Trappmann B, Gautrot J E, Connelly J T, Strange D G, Li Y, Oyen M L, Stuart M A C, Boehm H, Li $B$ and Vogel V 2012 Extracellular-matrix tethering regulates stem-cell fate Nat. Mater. 11642

[10] Tzvetkova-Chevolleau T, Stéphanou A, Fuard D, Ohayon J, Schiavone P and Tracqui P 2008 The motility of normal and cancer cells in response to the combined influence of the substrate rigidity and anisotropic microstructure Biomaterials 29 1541-51

[11] Leipzig N D and Shoichet M S 2009 The effect of substrate stiffness on adult neural stem cell behavior Biomaterials 30 6867-78

[12] Levental I, Georges P C and Janmey P A 2007 Soft biological materials and their impact on cell function Soft Matter 3 299-306

[13] Martinez J S, Lehaf A M, Schlenoff J B and Keller III T C 2013 Cell durotaxis on polyelectrolyte multilayers with photogenerated gradients of modulus Biomacromolecules 14 1311-20

[14] Seidi A, Ramalingam M, Elloumi-Hannachi I, Ostrovidov S and Khademhosseini A 2011 Gradient biomaterials for soft-to-hard interface tissue engineering Acta Biomater. 7 1441-51

[15] Kim H-D and Peyton S R 2012 Bio-inspired materials for parsing matrix physicochemical control of cell migration: a review Integr. Biol. 4 37-52

[16] Lo C-M, Wang H-B, Dembo M and Wang Y-I 2000 Cell movement is guided by the rigidity of the substrate Biophys. J. 79 144-52

[17] Cheung Y K, Azeloglu E U, Shiovitz D A, Costa K D, Seliktar D and Sia S K 2009 Microscale control of stiffness in a cell - adhesive substrate using microfluidics - based lithography Angew. Chem. Int. Ed. 48 7188-92

[18] Lv H, Wang H, Zhang Z, Yang W, Liu W, Li Y and Li L J L s 2017 Biomaterial stiffness determines stem cell fate 178 42-8

[19] Keselowsky B G, Collard D M and García A J 2005 Integrin binding specificity regulates biomaterial surface chemistry effects on cell differentiation Proc. Natl. Acad. Sci. U.S.A. 102 
5953-7

[20] Hadden W J, Young J L, Holle A W, McFetridge M L, Kim D Y, Wijesinghe P, Taylor-Weiner H, Wen J H, Lee A R, Bieback K, Vo B-N, Sampson D D, Kennedy B F, Spatz J P, Engler A J and Choi Y S 2017 Stem cell migration and mechanotransduction on linear stiffness gradient hydrogels Proc. Natl. Acad. Sci. U.S.A. 114 5647-52

[21] Li J, Zhang F, Yu L, Fujimoto N, Yoshioka M, Li X, Shi J, Kotera H, Liu L and Chen Y 2017 Culture substrates made of elastomeric micro-tripod arrays for long-term expansion of human pluripotent stem cells J. Mater. Chem. B 5 236-44

[22] Trichet L, Le Digabel J, Hawkins R J, Vedula S R K, Gupta M, Ribrault C, Hersen P, Voituriez R and Ladoux B 2012 Evidence of a large-scale mechanosensing mechanism for cellular adaptation to substrate stiffness Proc. Natl. Acad. Sci. U.S.A. 109 6933-8

[23] Wei J, Shi J, Wang B, Tang Y, Tu X, Roy E, Ladoux B and Chen Y 2016 Fabrication of adjacent micropillar arrays with different heights for cell studies Microelectron. Eng. 158 22-5

[24] Sochol R D, Higa A T, Janairo R R, Li S and Lin L 2011 Unidirectional mechanical cellular stimuli via micropost array gradients Soft Matter 7 4606-9

[25] Lee S, Hong J and Lee J 2016 Cell motility regulation on a stepped micro pillar array device (SMPAD) with a discrete stiffness gradient Soft Matter 12 2325-33

[26] Saez A, Buguin A, Silberzan P and Ladoux B 2005 Is the mechanical activity of epithelial cells controlled by deformations or forces? Biophys. J. 89 L52-L4

[27] Saez A, Anon E, Ghibaudo M, du Roure O, Di Meglio J, Hersen P, Silberzan P, Buguin A and Ladoux B 2010 Traction forces exerted by epithelial cell sheets J. Phys.: Condens. Matter 22 194119

[28] Roca-Cusachs P, Sunyer R and Trepat X 2013 Mechanical guidance of cell migration: lessons from chemotaxis Curr. Opin. Cell Biol. 25 543-9

[29] Rotsch C, Jacobson K and Radmacher M 1999 Dimensional and mechanical dynamics of active and stable edges in motile fibroblasts investigated by using atomic force microscopy Proc. Natl. Acad. Sci. U.S.A. 96 921-6

[30] Gupta M, Sarangi B R, Deschamps J, Nematbakhsh Y, Callan-Jones A, Margadant F, Mège R-M, Lim C T, Voituriez R and Ladoux B 2015 Adaptive rheology and ordering of cell cytoskeleton govern matrix rigidity sensing Nat. Commun. 67525

[31] Yang L, Yangben Y and Chiang M 2011 Cell Morphology Linked to Substrate Stiffness-A Possible Solution to Determine the Cell Modulus Biophys. J. 100 157a

[32] Choi M J, Park J Y, Cha K J, Rhie J-W, Cho D-W and Kim D S 2012 Micropattern array with gradient size ( $\mu$ PAGS) plastic surfaces fabricated by PDMS (polydimethylsiloxane) mold-based hot embossing technique for investigation of cell-surface interaction Biofabrication 4045006

[33] Duclos G, Erlenkämper C, Joanny J-F and Silberzan P J N P 2017 Topological defects in confined populations of spindle-shaped cells 1358

[34] Keber F C, Loiseau E, Sanchez T, DeCamp S J, Giomi L, Bowick M J, Marchetti M C, Dogic Z and Bausch A R J S 2014 Topology and dynamics of active nematic vesicles 345 1135-9

[35] Cai Y, Rossier O, Gauthier N C, Biais N, Fardin M-A, Zhang X, Miller L W, Ladoux B, Cornish V W and Sheetz M P 2010 Cytoskeletal coherence requires myosin-IIA contractility J. Cell Sci. jcs. 058297 
[36] Delanoe-Ayari H, Al Kurdi R, Vallade M, Gulino-Debrac D and Riveline D 2004 Membrane and acto-myosin tension promote clustering of adhesion proteins Proc. Natl. Acad. Sci. U.S.A. 101 2229-34

[37] Solon J, Levental I, Sengupta K, Georges P C and Janmey P A 2007 Fibroblast adaptation and stiffness matching to soft elastic substrates Biophys. J. 93 4453-61

[38] Sajeesh P, Raj A, Doble M and Sen A 2016 Characterization and sorting of cells based on stiffness contrast in a microfluidic channel Rsc Advances 6 74704-14

[39] Kuo C H R, Xian J, Brenton J D, Franze K and Sivaniah E J A m 2012 Complex stiffness gradient substrates for studying mechanotactic cell migration 24 6059-64

[40] Sun Y, Yong K M A, Villa-Diaz L G, Zhang X, Chen W, Philson R, Weng S, Xu H, Krebsbach P H and Fu J 2014 Hippo/YAP-mediated rigidity-dependent motor neuron differentiation of human pluripotent stem cells Nat. Mater. 13599

[41] Tang Y, Liu L, Li J, Yu L, Wang L, Shi J and Chen Y 2016 Induction and differentiation of human induced pluripotent stem cells into functional cardiomyocytes on a compartmented monolayer of gelatin nanofibers Nanoscale 8 14530-40

[42] Yukawa H, Noguchi $\mathrm{H}$ and Hayashi S 2011 Embryonic body formation using the tapered soft stencil for cluster culture device Biomaterials 32 3729-38

[43] Wang B, Tu X, Wei J, Wang L and Chen Y 2018 Substrate elasticity dependent colony formation and cardiac differentiation of human induced pluripotent stem cells Biofabrication 11015005 


\section{Figure and captions}

Figure 1. (a) Schematic illustration of cell culture on micropillar array with height gradient. (b, c) Scanning electron microscope (SEM) images of PDMS micropillars of height gradient with period 160 $\mu \mathrm{m}(\mathrm{P} 160)$ and $310 \mu \mathrm{m}$ (P310), respectively. (d) Variation of the pillar height as a function of pillar position for samples $\mathrm{P} 160$ and $\mathrm{P} 310$.

Figure 2. Microfluidic device for pillars elasticity demonstration. (a) Schematic diagram of gradient micropillars integrated into microfluidic chip for elasticity demonstration. (b, d) and (c, e) phase contrast images of one period $(160 \mu \mathrm{m}, 310 \mu \mathrm{m})$ of gradient pillars in channel before and after deformation. (f, h) The displacement of pillars of P160 and P310 in microfluidic channel. Pillar position represents every single pillar from shortest to highest one in half of the period if we assume the shortest pillar started from position 0. (g, h) Calculated effective Young's modules of samples P160 and P310.

Figure 3. (a) Time-lapse images of NIH $3 \mathrm{~T} 3$ cells showing migratory behaviors on PDMS pillar arrays with height gradient of sample P160. Short pillars were in brighter area as indicated in orange box; (b,

c) Phase contrast images showing cell elongation and localization on PDMS pillar arrays of samples P160 and P310 after $24 \mathrm{~h}$ incubation; (d, e) NIH 3 T3 cells migration distance parallel and perpendicular to the elasticity gradient of samples P160 and P310; (f) Cells migration velocity on PDMS pillar arrays with different gradient strengths. $\mathrm{n}=14 . * \mathrm{P}<0.05, * * \mathrm{P}<0.01, * * * \mathrm{P}<0.001$.

Figure 4. Immunofluorescence images of NIH 3 T3 cells after 1 day (a-c) and 4 days (g-i) incubation on flat, P160 and P310 PDMS samples. Cell nuclei were stained by DAPI (blue) and actin cytoskeletal networks in green (Alexa Fluor 488 phalloidin). Distribution of alignment of cells after 1 day (d-f) and 4 days (j-1) incubation on flat PDMS, P160 and P310 samples. $\mathrm{n}>80$.

Figure 5. Immunofluorescence and SEM images of high magnitude of NIH 3T3 cells after 1 day 
incubation on (a, c) P160 and (b, d) P310. (e, f) Cell and nucleus area on the three types of substrates after 1 day incubation; (g, h) Substrate elasticity gradient induced shape anisotropy of cytoskeleton and nucleus (ratio of the length to the width for cells and their nuclei). $\mathrm{n}=40, * \mathrm{P}<0.05, * * \mathrm{P}<0.01$, $* * * \mathrm{P}<0.001$.

Figure 6. (a, b) SEM images of PDMS pillar arrays with ripple-like elasticity gradient (sample R160); (c) Calculated effective Young's modules of PDMS pillar arrays with ripple-like elasticity gradient; (d-f) Immunofluorescence images of NIH 3T3 cells after 1 day, 3 days and 4 days incubation on sample R160. Cell nuclei were stained by DAPI (blue) and actin cytoskeletal networks in green (Alexa Fluor 488 phalloidin).

Figure 7. (a) iPSCs cultured on P160 at day 0, day 1 and day2. (b) Immunofluorescence images of iPSCs on P160 and flat PDMS substrates after culture 2 days (red: OCT4, green: SSEA4, blue: DAPI). (c, d) Box plot showing the positive OCT4 and SSEA4 cells on micropillar substrates and flat PDMS. 
Figure 1
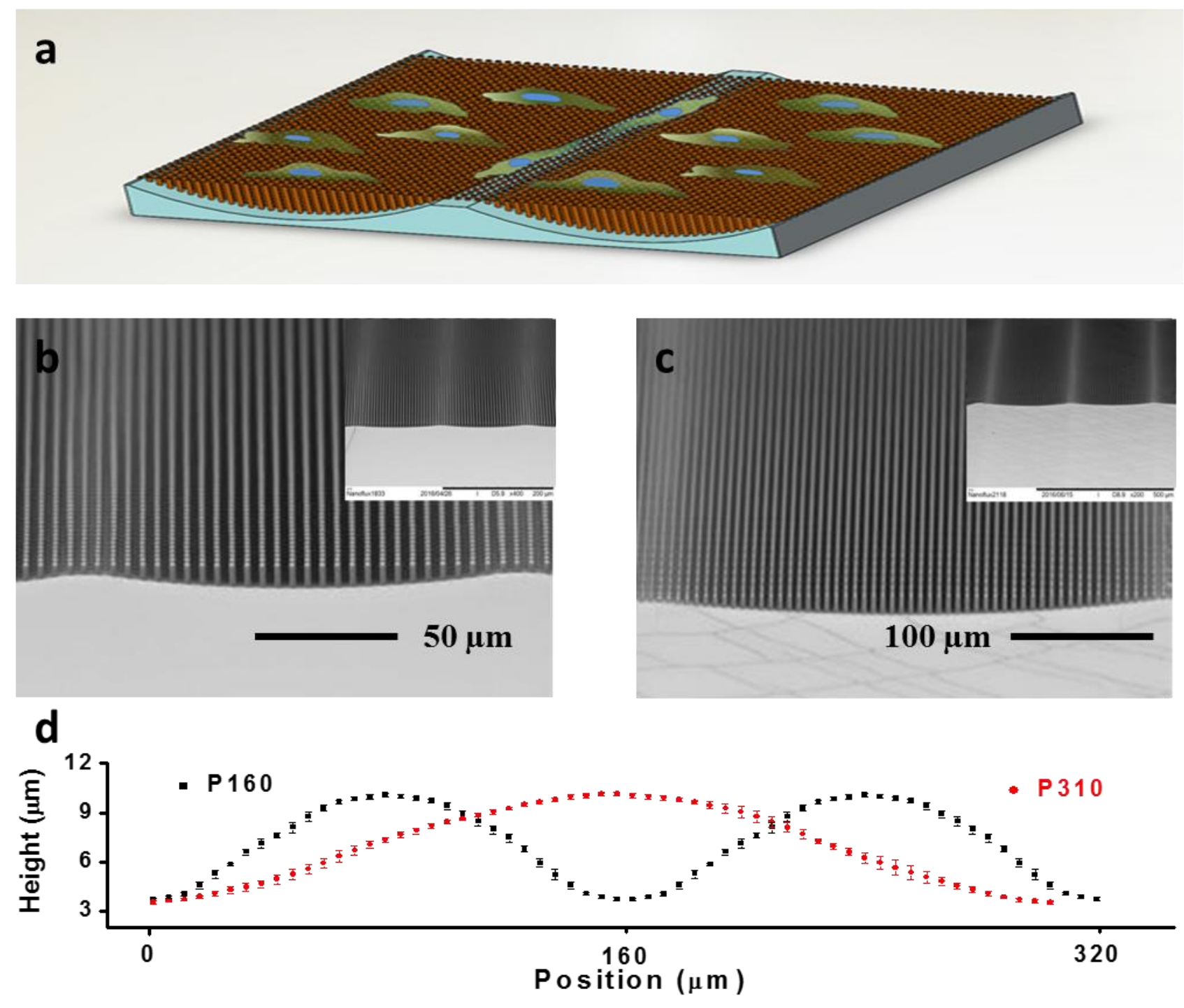
Figure 2
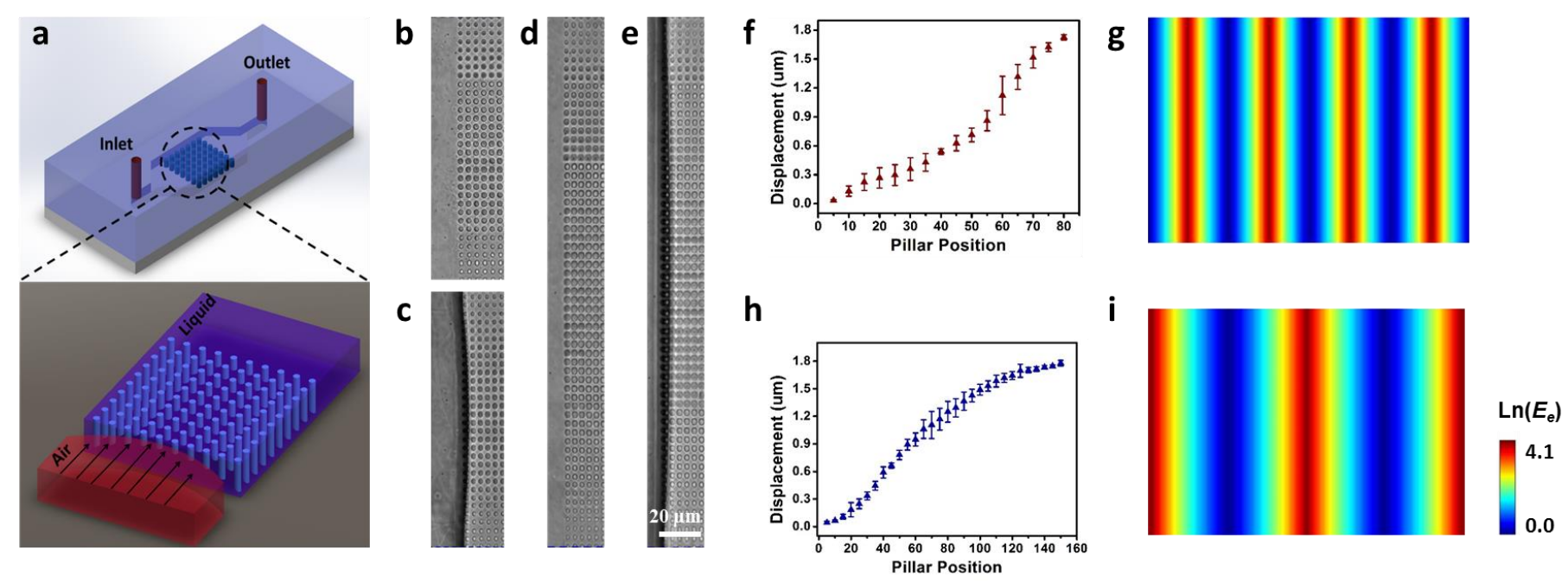
Figure 3
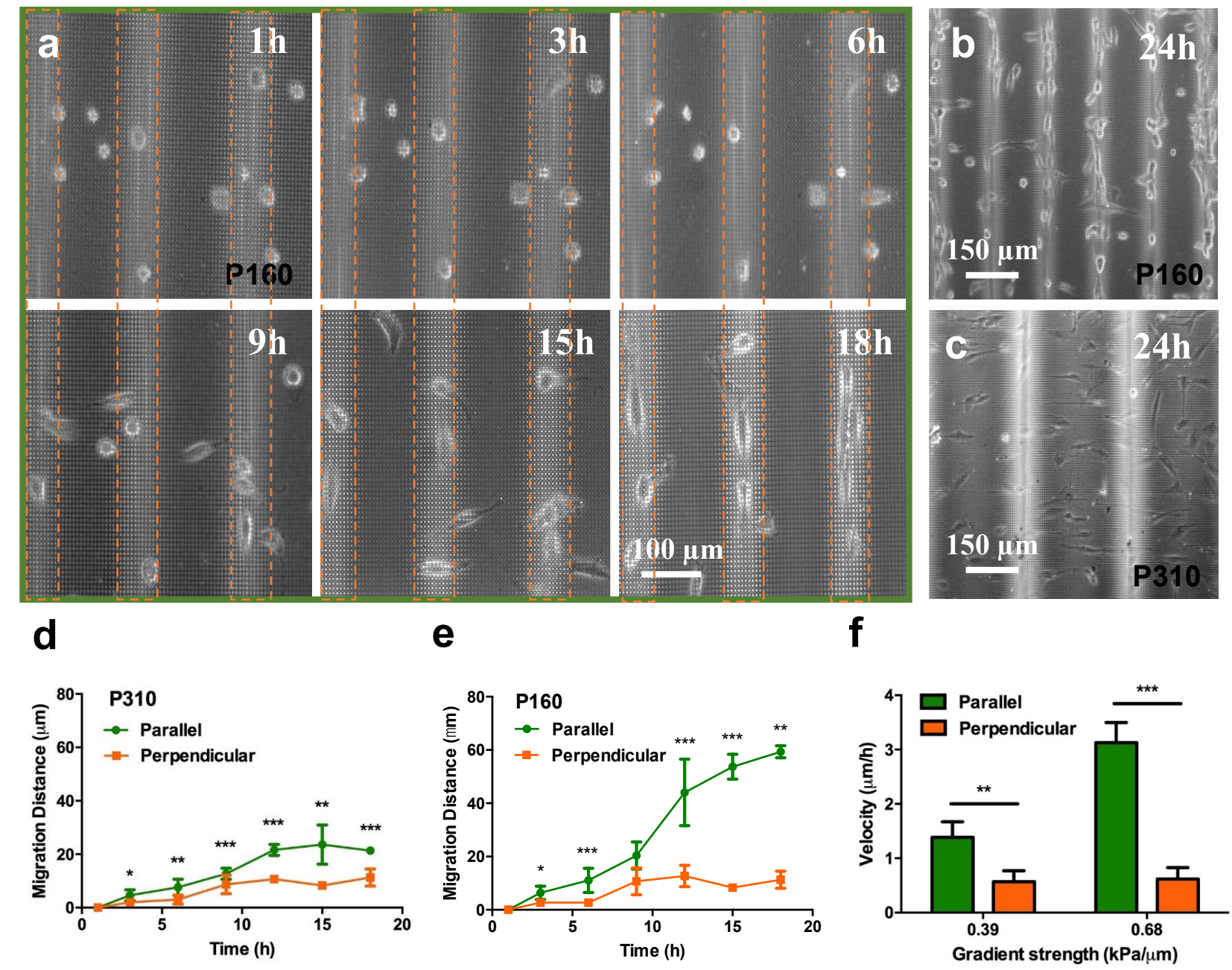
Figure 4
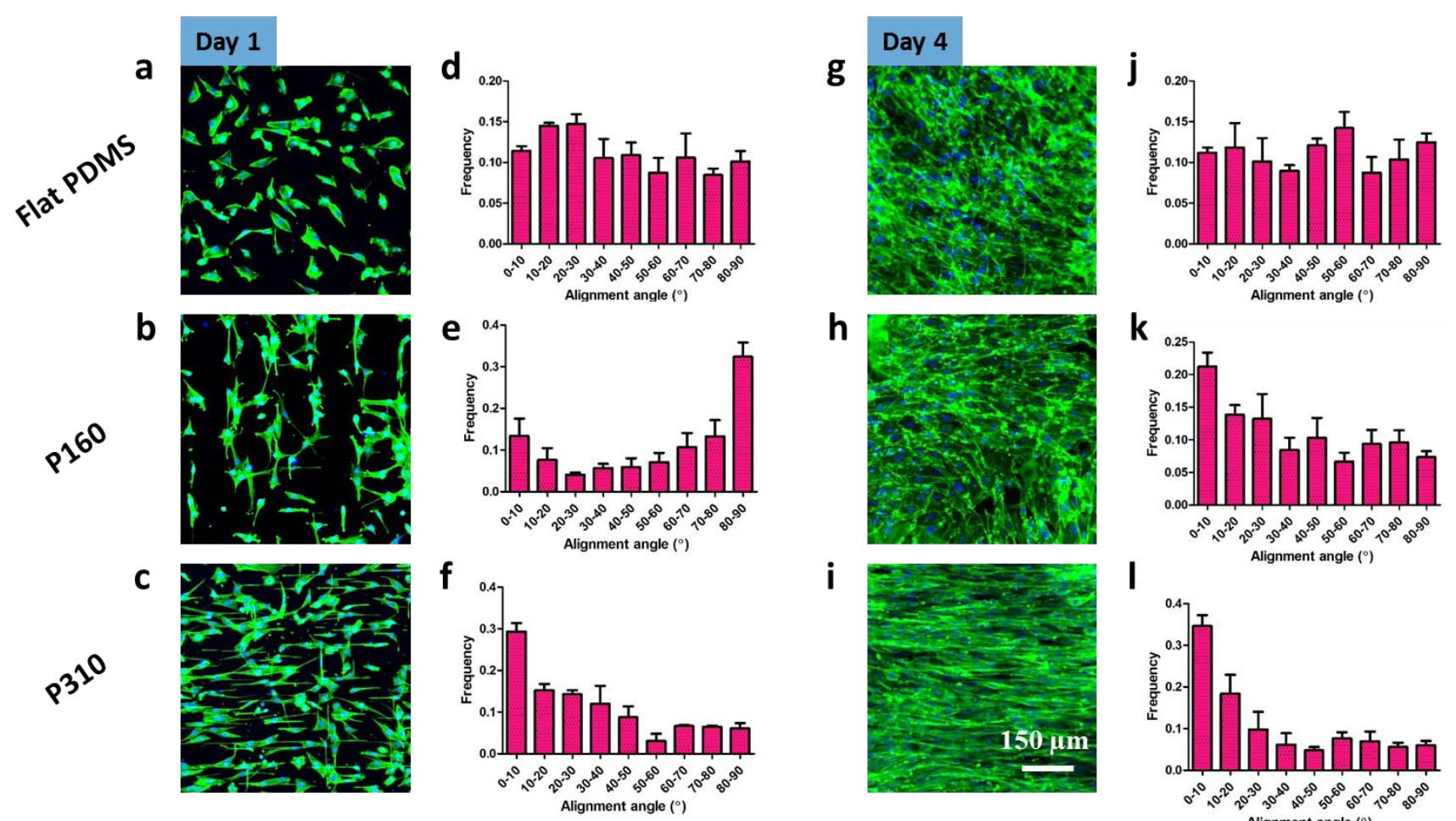

i
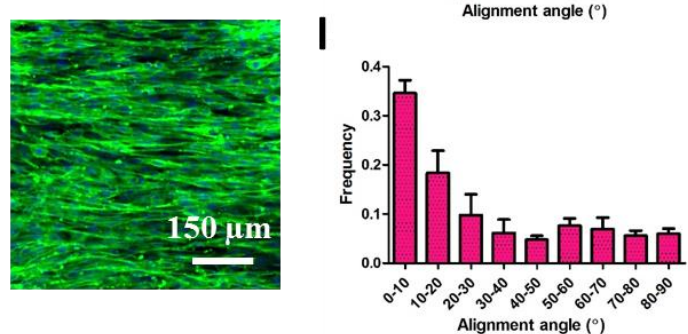
Figure 5
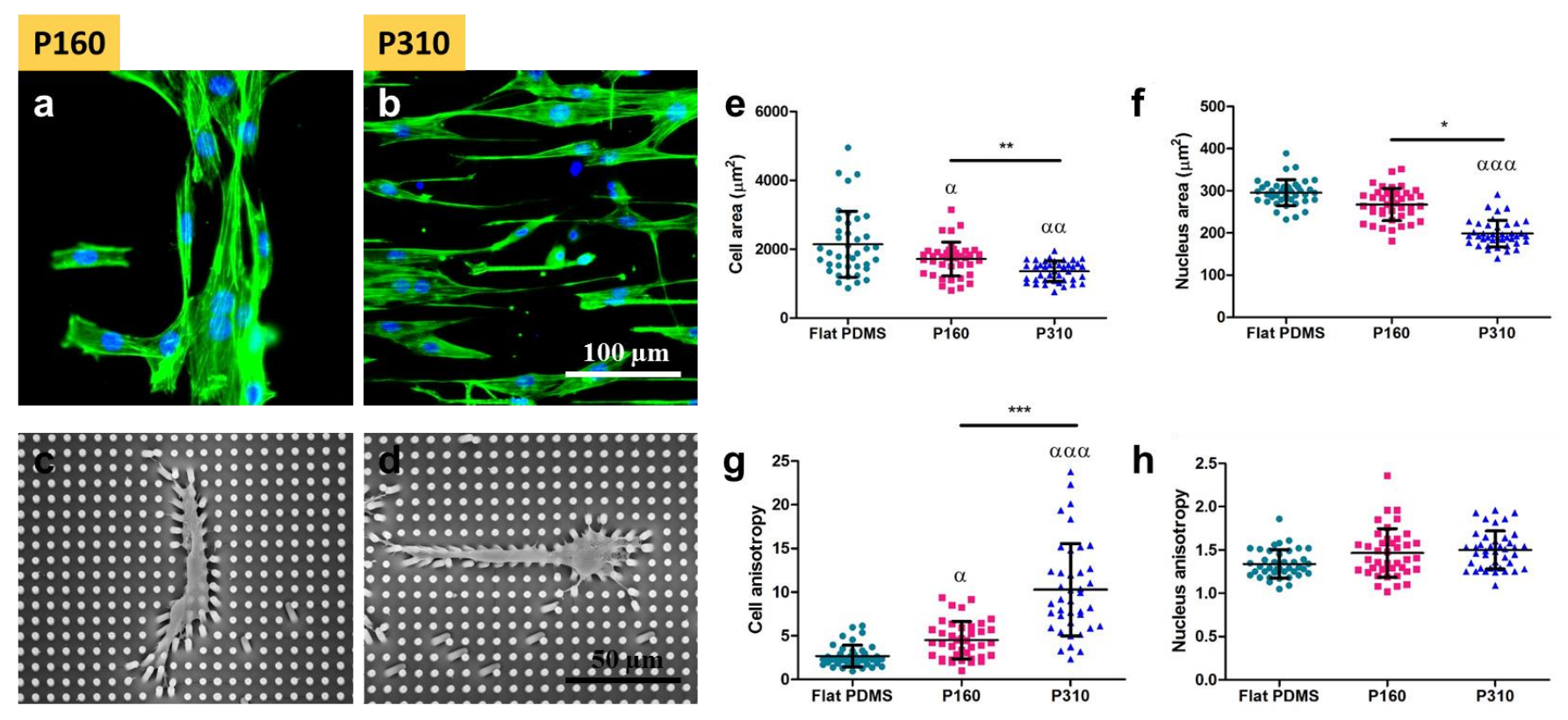
Figure 6
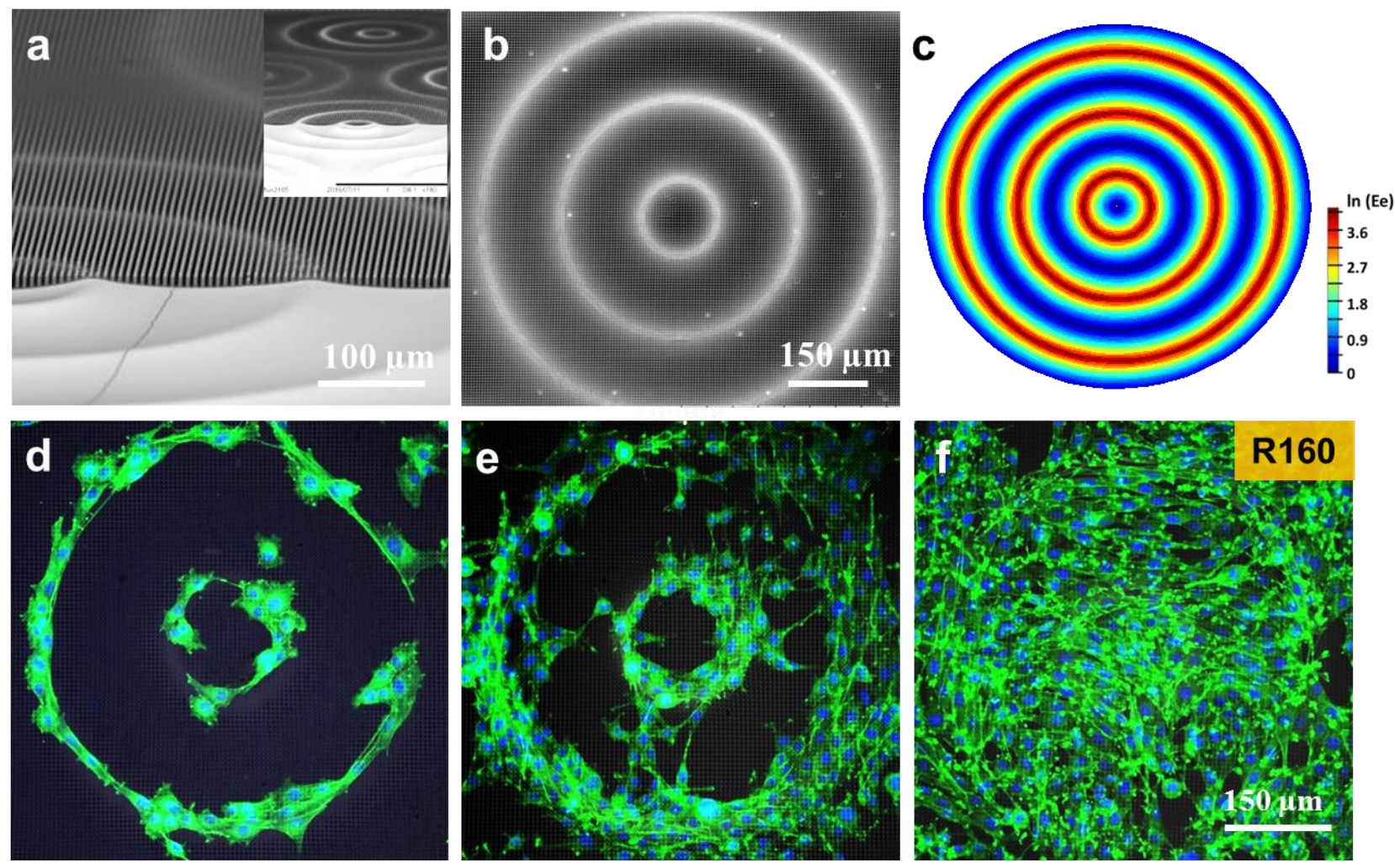
Figure 7
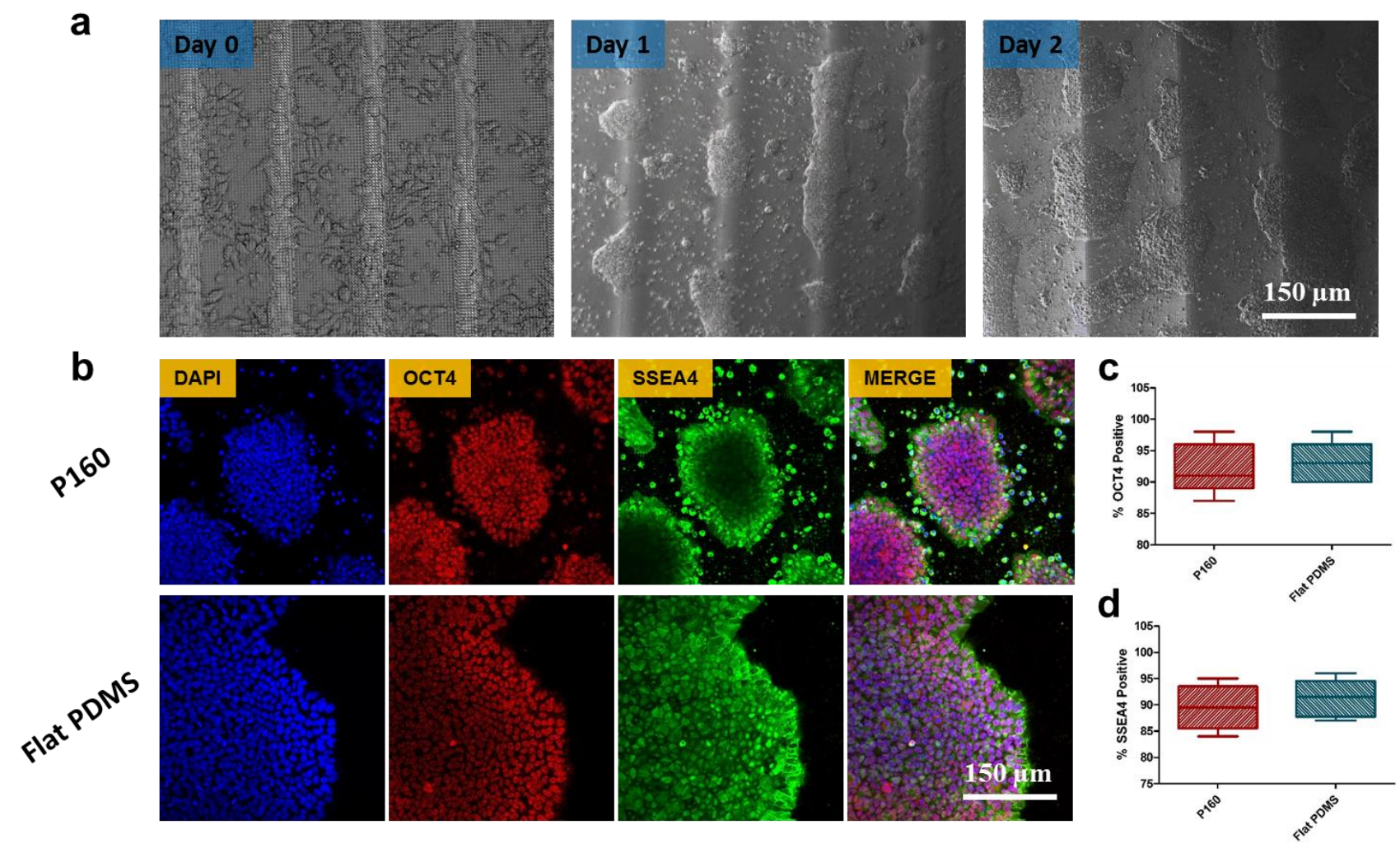


\title{
Support Information
}

Fabrication of elastomer pillar arrays with elasticity gradient for cells migration, elongation and patterning

\author{
Bin Wang ${ }^{\mathrm{a}}$, Jin Wei ${ }^{\mathrm{a}}$, Xiaolong $\mathrm{Tu}^{\mathrm{a}}{ }^{\mathrm{a}}$, Jian Shi ${ }^{\mathrm{a}}$, Yong Chen ${ }^{\mathrm{a}, \mathrm{b}^{*}}$ \\ ${ }^{a}$ PASTEUR, Département de chimie, école normale supérieure, PSL \\ University, Sorbonne Université, CNRS, 75005 Paris, France \\ ${ }^{b}$ Institute for interdisciplinary research center, Jianghan University, Wuhan 430056, China \\ * Corresponding author. Email: yong.chen@ens.fr (Y. Chen)
}


Supplementary Figure S1. Schematic illustration of fabrication of PDMS micropillar arrays with height gradient. Cr mask with dot arrays was firstly obtained from direct laser writing. Then first SU8 photoresist was spin-coated and uv exposed with another pre-patterned mask, followed by developing to obtain stripes pattern. After that, second thin layer of SU-8 resist was spin-coated and exposed to form wavy features on which spin-coating a third layer of SU-8 resist and backside expose to obtain gradient micropillars in photoresist. After two times of soft replication, PDMS micropillars with height gradient obtained.

Supplementary Figure S2. Phase contrast image of gradient pillars deformation in different period.

Supplementary Figure S3. Phase contrast image of Hela cells after 1 day incubation on sample P160, showing neither elongation nor migration caused by elasticity gradient of PDMS pillars.

Supplementary Figure S4. Phase contrast image of NIH 3T3 cells after 1 day incubation on micropillars with uniform height of $5 \mu \mathrm{m}$.

Supplementary Video S1. Time-lapse observation of NIH 3T3 cell migration on micropillar substrate with gradient period $160 \mu \mathrm{m}$.

Supplementary Video S2. Video of micropillars in microfluidic chamber with air and water flow. 


\section{Supplementary Figure S1}

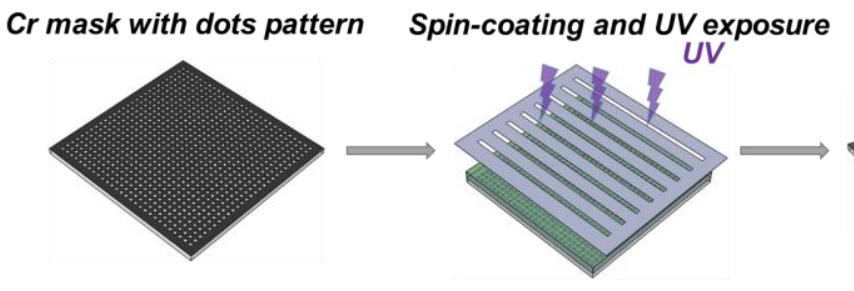

Development

$2^{\text {nd }}$ spin-coating to form wave pattern

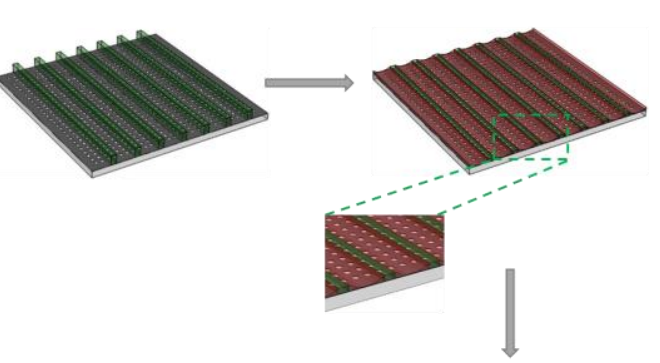

PDMS gradient pillar

Negative PDMS mould

Develop gradient pillars

$3^{\text {rd }}$ spin-coating and backside expose
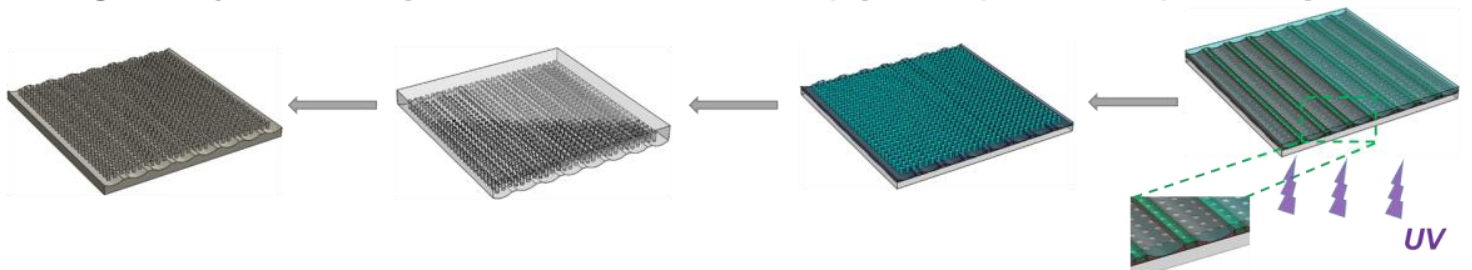
Supplementary Figure S2

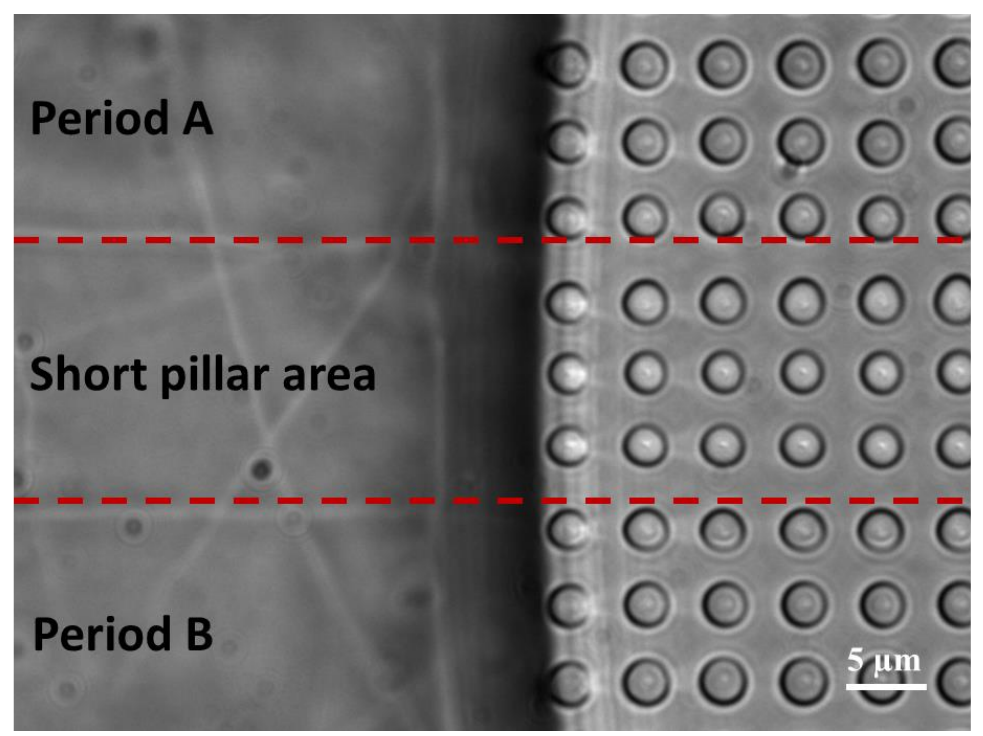


Supplementary Figure S3

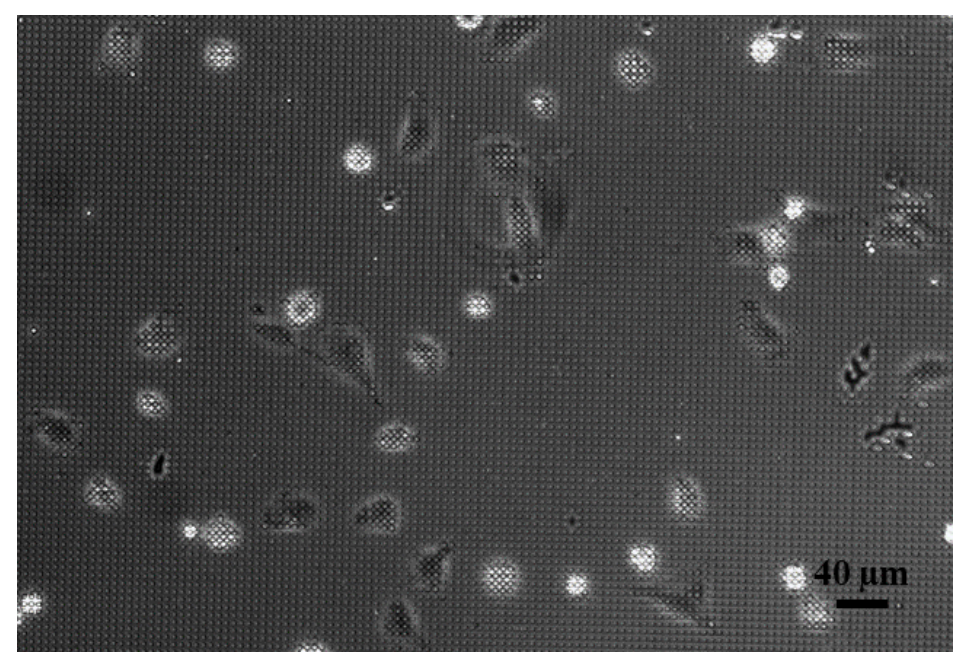


Supplementary Figure S4

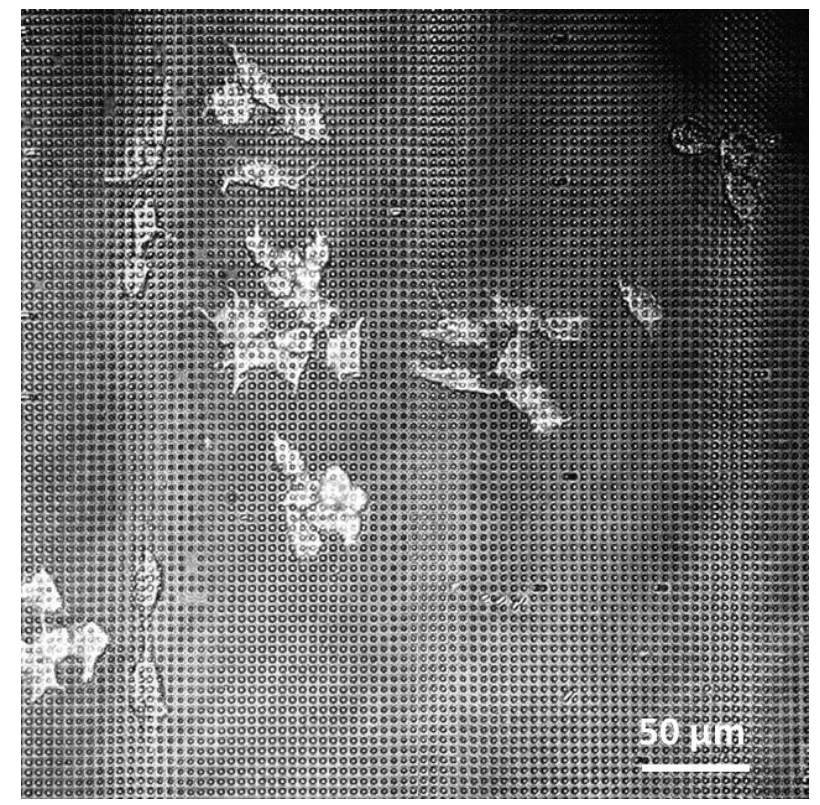

